\title{
Innovative technology for reducing the rate of sediment formation in the power plants channels
}

\author{
Anatoly Muraviev ${ }^{1}$, Alexander Nadeev $^{1}$, Alexander Naumov $^{1, *}$, Inna Pereslavtseva ${ }^{1}$ \\ ${ }^{1}$ Voronezh State Technical University, 14, Moscow Avenue, 394026, Voronezh, Russia
}

\begin{abstract}
This paper considers the increasing the mass transfer intensity due to the use of annular baffles in the tubes of shell-and-tube heat exchange apparatuses. A mathematical model for calculating the system of equations for the evolution of particle distribution in the form of sediments has been developed.
\end{abstract}

\section{Introduction}

Currently, one of the main problems at energy-oriented companies is the various sediments formation in heat exchangers. Sediments on heat transfer surfaces adversely affect hydrodynamics and heat transfer, which leads to an increase in financial costs during the apparatus operation and an efficiency decrease, as well as additional costs for repair and maintenance of equipment.

One of the most effective ways to reduce the sediments intensity on the heat transfer surfaces is the use of artificial turbulization of the heat carrier stream [1]. The essence of this method is that knurling of annular baffles is performed on the working surface of the heat exchanger tubes along their entire length, after a certain step [2]. As a result of knurling the grooves are formed both from the outside and inside the tube, due to which the flow swirls. The fluid flow at the wall of the tube, where its velocity is the smallest, begins to turbulize. Thus, a more efficient entrainment of solid particles occurs, thereby reducing the possibility of sediment formation. The methods of dealing with sediments are presented on Figure 1 [3].

\footnotetext{
* Corresponding author: nix2001@yandex.ru
} 


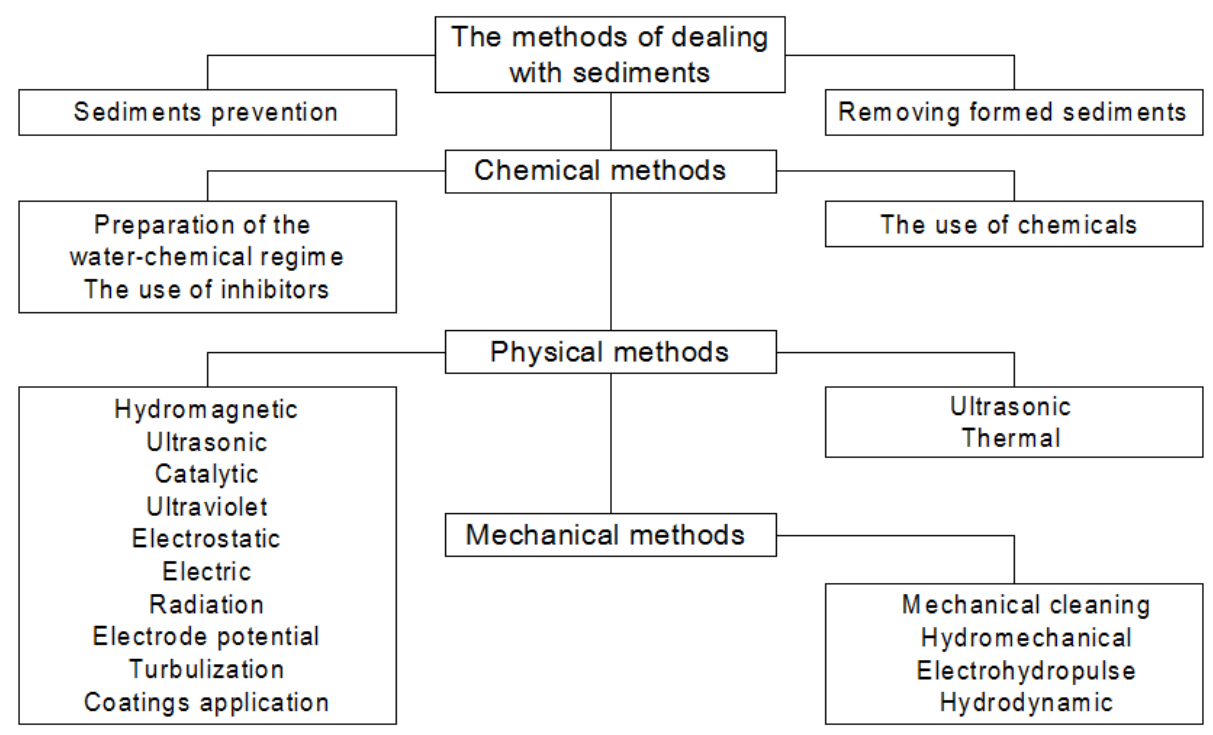

Fig. 1. The methods of dealing with sediments.

\section{Design features of heat exchangers}

Two experimental shell-and-tube heat exchangers have been designed in order to study the influence of the coolant flow regime on the sediment formation process (Figure. 1). The studied tubes with various geometric characteristics were placed in them (Figure 2). The number of tubes in each device is 7 pieces. The saturated steam with a pressure of $P=6$ atm. and temperature $\mathrm{t}=158^{\circ} \mathrm{C}$ was used as a heating medium (annulus). The water with an inlet temperature of $\mathrm{t}=20^{\circ} \mathrm{C}$, Ca stiffness $-2.4 \mathrm{ml} /$ equiv $/ \mathrm{dm} 3$, and general stiffness. $-2.5 \mathrm{ml} /$ equiv / dm3 was used as a heated medium (tube space). The diagram of the heat exchanger is shown are presented on Figure 2. The experimental tube is shown in Figure 3. 


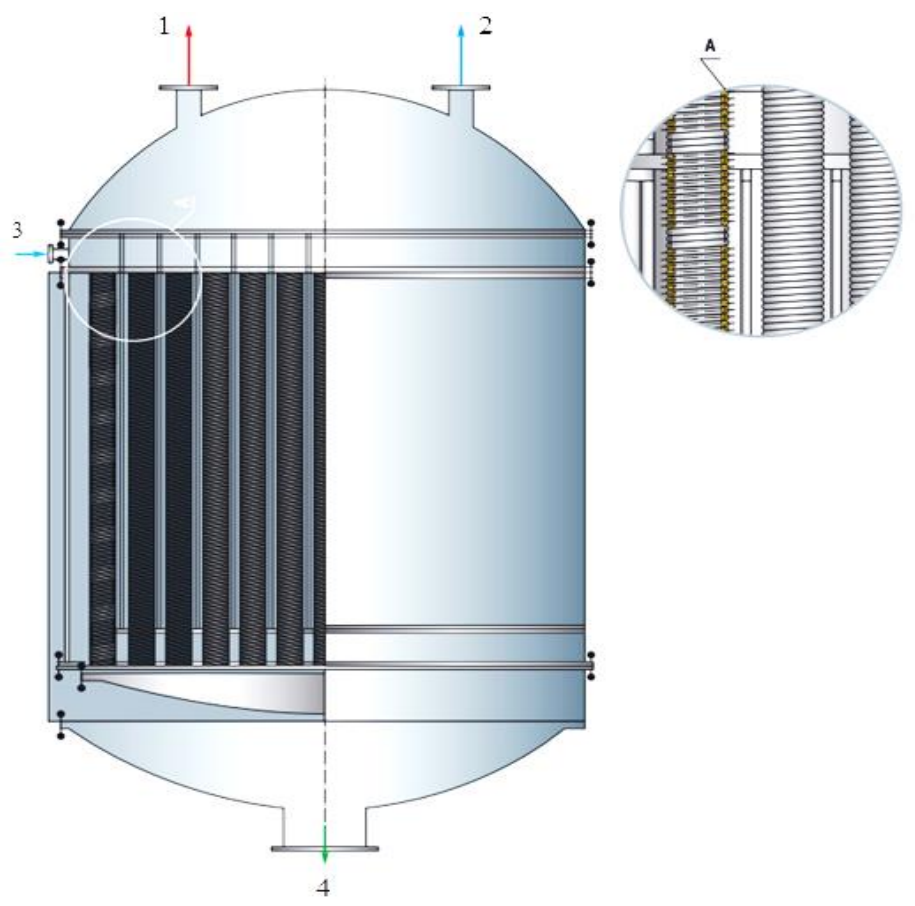

Fig. 2. Heat exchanging apparatus design: 1 - hot water; 2 - cold water; 3 - vapour; 4 - condensate.

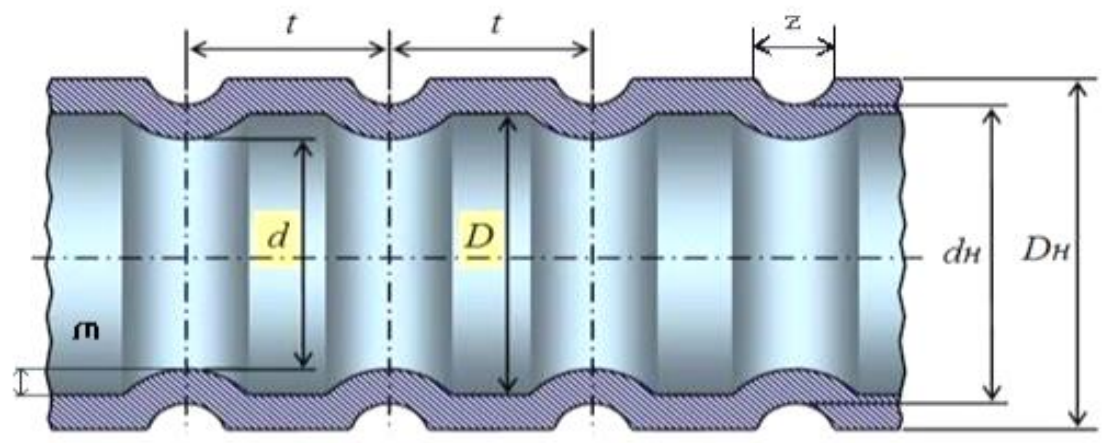

Fig. 3. Longitudinal section of the tube.

Characteristics of the experimental tubes are presented in Table. 1. External diameter of tubes $D=19 \mathrm{~mm}$. Internal diameter of tubes $d=16 \mathrm{~mm}$. Tube wall thickness $S=1,5$ $\mathrm{mm}$.

Since the material of the tubes can also have a chemical effect on the sediment formation, some tubes were made of $1 \times 18 \mathrm{~h} 10$ stainless steel, and some of the Lo-70 brass [4]. Two tubes were smooth, and five had annular diaphragms. 
Table 1. Characteristics of tubes.

\begin{tabular}{|c|c|c|c|c|c|c|}
\hline $\begin{array}{c}\text { Test } \\
\text { tube. } \\
\text { № }\end{array}$ & $\begin{array}{c}\text { Tube } \\
\text { material } \\
D_{H} \cdot \mathbf{m m}\end{array}$ & $\begin{array}{c}\text { Turbulator } \\
\text { Step } \\
t . \mathbf{m m}\end{array}$ & $\begin{array}{c}\text { Inner } \\
\text { diameter of } \\
\text { diaphragms } \\
d \cdot \mathbf{m m}\end{array}$ & $\begin{array}{c}\text { Groove depth of } \\
\text { turbulator } \\
m \cdot \mathbf{m m}\end{array}$ & $d / D$ & $t / D$ \\
\hline 1 & $1 \mathrm{x} 18 \mathrm{~h} 10$ & - & - & - & - & - \\
\hline 2 & Lo-70 & - & - & - & - & - \\
\hline 3 & Lo-70 & 2 & 14 & 1 & 0.875 & 0.125 \\
\hline 4 & $1 \mathrm{x} 18 \mathrm{~h} 10$ & 4 & 15 & 0.5 & 0.937 & 0.25 \\
\hline 5 & Lo-70 & 4 & 14 & 1 & 0.875 & 0.25 \\
\hline 6 & $1 \mathrm{x} 18 \mathrm{~h} 10$ & 4 & 12 & 2 & 0.75 & 0.25 \\
\hline 7 & Lo-70 & 8 & 14 & 1 & 0.875 & 0.5 \\
\hline
\end{tabular}

\section{Experimental research on a heat exchange apparatus}

An experimental study of heat exchangers was carried out for 2000 hours. This duration allows you to record the change in the intensity of heat and mass transfer and hydraulic resistance, depending on the heated fluid speed and the turbulators characteristics [5]. Due to the fact that the coolant speed directly affects the sediment formation, two heat exchangers were used in the experiment. One device was operated in laminar mode of operation, and the second in turbulent mode. The results of the local sediments distribution studies are presented in Figure 4 and Table. 2.
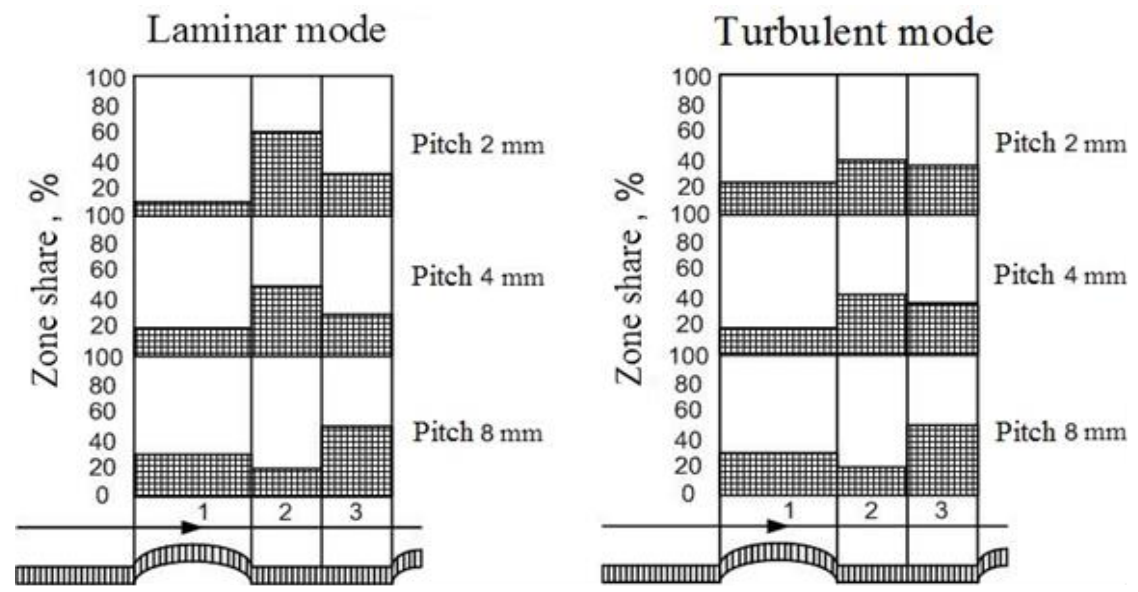

Fig. 3. Percent sediment.

Table 2. Research result.

\begin{tabular}{|c|c|c|c|c|c|c|}
\hline \multirow[t]{2}{*}{$\begin{array}{l}\text { Sample } \\
\text { tubes. } \\
\text { № }\end{array}$} & \multicolumn{3}{|c|}{$\begin{array}{c}\text { Laminar mode } \\
\text { Sediment thickness } \delta_{H} \cdot \mathrm{mm}\end{array}$} & \multicolumn{3}{|c|}{$\begin{array}{c}\text { Turbulent mode } \\
\text { Sediment thickness } \delta_{H} . \mathrm{mm}\end{array}$} \\
\hline & zone 1 & zone 2 & zone 3 & zone 1 & zone 2 & zone 3 \\
\hline 1 & \multicolumn{3}{|c|}{0.56} & \multicolumn{3}{|c|}{0.89} \\
\hline 2 & \multicolumn{3}{|c|}{0.59} & \multicolumn{3}{|c|}{0.91} \\
\hline 3 & 0.78 & 0.86 & 0.82 & 0.49 & 0.57 & 0.56 \\
\hline 4 & 0.82 & 0.87 & 0.84 & 0.49 & 0.55 & 0.52 \\
\hline 5 & 0.83 & 0.86 & 0.84 & 0.48 & 0.54 & 0.51 \\
\hline 6 & 0.81 & 0.85 & 0.83 & 0.48 & 0.53 & 0.5 \\
\hline 7 & 0.83 & 0.81 & 0.86 & 0.53 & 0.49 & 0.56 \\
\hline
\end{tabular}


The experimental results showed that the use of tubes with turbulators during the heat exchanger operation of in the laminar regime of the coolant flow is not effective, since the tubes were subject to greater sediment fouling on the heat transfer surfaces. In turn, the use of tubes with turbulators in a turbulent mode led to a decrease in the intensity of the sediment formation on their surface.

So, the minimum rate of sediment formation in the form of deposits was observed for pipes with turbulators with a turbulent coolant flow regime at a ratio $m / d=4 \cdot 103-14 \cdot 103$ and $\mathrm{t} / \mathrm{m}=1.0-1.8$, and with a further increase in the ratio $\mathrm{t} / \mathrm{m}$ the mass transfer is reduced. In the sample No 6, the amount of sediment was the lowest with a ratio of $d / D=0,75$ and $t / D=0,25$. Thus, the experiments carried out have confirmed that in the tubes with turbulizer the amount of sediments is much less than in smooth pipes. Their formation obeys the asymptotic law, but only under the condition of the coolant turbulent flow. It should be noted that almost all heat exchangers operate with turbulent movement of the coolant, which confirms the relevance of their use. The use of knurled pipes in heat exchangers increases the exploitation period, improves heat transfer, and leads to an increase in heat transfer intensification. The knurling of one tube costs from 2 to $4 \%$ of the pipe cost.

\section{Mathematical model}

The system of equations for the evolution of the sediment particles distribution has the form:

$$
\begin{aligned}
\left(\frac{d N_{k}}{d t}\right) & =\delta_{K, 0}\left(\frac{d N_{0}}{d t}\right)_{\text {of the beginning }}+\left(\frac{d N_{K}}{d t}\right)_{\text {of the development }}+ \\
& \left(\frac{d N_{K}}{d t}\right)_{\text {of the fusion }}+\left(\frac{d N_{K}}{d t}\right)_{\text {of the sedimentation }}
\end{aligned}
$$

where $\delta_{K, m}$ - the Kronecker symbol.

We write the equation of mass balance, taking into account the decrease in concentration due to the crystals excretion $\mathrm{CaCO}_{3}$ [6]. Thus, a decrease in concentration $C$ occurs due to crystal growth:

$$
\left(\frac{d C}{d t}\right)=-(\rho) V(S(T, t), T) \sigma_{\text {full }},
$$

where $\rho$ is the crystal concentration (molar) $\mathrm{CaCO}_{3}$;

$\sigma_{\text {full }}$ is the surface of all crystals (whole) in the volume $\Delta V$ :

$$
\sigma_{\text {full }}=\xi \sum_{K+1}^{M} N_{K}\left(d_{K}^{\sigma}\right)^{2},
$$

where $\xi$ is the shape factor (crystal shape): 


$$
\xi=\frac{\sigma_{\text {crystal's }}}{d^{2}}
$$

$\sigma_{\text {crystal's }}$ is the surface area of the sediment crystal (with the diameter $d$ ).

Thus, the equation for concentration will have the form:

$$
\frac{d C}{d t}=-\rho V(S, T) \xi \sum_{m=1}^{M}\left(d_{K}^{\sigma}\right)^{2} N_{K} .
$$

The concentration $C$ is expressed in moles per unit volume of solution, similar to the values $N_{K}$. Formulas (1) - (5) will have a closed model of the studied sediment formation process.

The general formulation of the equations includes boundary initial conditions and their formulation. In this stationary model the systems are depicted in a spatially distributed plane and the boundary conditions are taken out in the Euler coordinates to calculate the coolant temperature distribution [7]. Thus, due to the stationary nature of the problem, the initial conditions are absent, and the initial conditions are specified in the Lagrange coordinates, since the coefficients of the variables depend on time. To do this, we use the boundary conditions in Euler coordinates, which corresponds to the initial conditions in the coordinates of the Lagrange boundary conditions [8]. The initial condition for the solution is the initial input concentration of $\mathrm{CaCO} 3$ water in the system at the inlet: $C(t)=C_{0}$. We set the initial conditions of the particles which, in fact, are determined by their distribution in the system and the state of the solution at the inlet. At the initial stage we can assume that the water does not contain $\mathrm{CaCO} 3$ crystal s[9]:

$$
N_{K 0}=0
$$

However, we can clearly say that, in reality, in water there is always a certain amount of particles already present in the system. Also the water pre-heating can occur in the water treatment system, and thus the formation of a certain initial distribution [10]:

$$
N_{K}(0)=N_{K}^{0}
$$

This distribution can be obtained provided that the time at a given temperature under initial conditions is taken into account (6). Thus, as a result, the distribution of particles is considered according to the initial conditions of the system (7).

\section{References}

1. A.V. Muravev, N.V. Mozgovoj, I.G. Drozdov, Bulletin of the Voronezh State Technical University 3(6), 33-38 (2007)

2. A.V. Muraviev, I.L. Bataronov, Bulletin of the Voronezh State Technical University 11(4), 111-114 (2015)

3. A.V. Muraviev, Hydrogasdynamics (practical application) textbook (Voronezh State Technical University, Voronezh, 2012)

4. Y.V. Balaban-Irmenin, Thermal Engineering 7, 43-47 (1998)

5. G.P. Sheldon, J. Materials for Energy System 5(4), 259-264 (1984) 
6. L. Dubin, R.L. Dammeier, R.A. Hart, Materials Perfomance 24(10), 27-33 (1985)

7. Z.L. Miropolsky, I.A. Bublikov, B.E. Novikov, Thermal engineering 5, 71-74 (1992)

8. G.A. Dreitser, Heat power engineering 3, 30-35 (1996)

9. V.I. Sharapov, M.A. Krylova, Thermal engineering 8, 47-49 (1996)

10. Z. Adamczyk, T.G.M.Van de Ven, Journal of Colloid and Interface Science 97(1), 6890 (1984)

11. Y.V. Dubenko, D.A. Gura, E.E. Dyshkant, International Multi-Conference on Industrial Engineering and Modern Technologies, FarEastCon 2019, 8934179 (2019). DOI: 10.1109/FarEastCon.2019.8934179

12. M. Kuzyakina, D. Gura, A. Sekisov, N. Granik, Advances in Intelligent Systems and Computing 983, 403-416 (2019). DOI: 10.1007/978-3-030-19868-8_41 